\title{
Volgarizzare i documenti, volgarizzare gli statuti nella Toscana tra Due e Trecento
}

\begin{abstract}
Il saggio si concentra sull'impiego del volgare in ambito documentario per la trasmissione di testi normativi (in particolare gli statuti) tra il XIII e il XIV secolo. L'autore rileva in tal senso le ragioni alla base dell'adozione della lingua vernacolare in specifici contesti politici e sociali della Toscana due-trecentesca (in primo luogo a Siena, poi a Pisa e a Firenze), e l'importanza assunta nel processo di istituzionalizzazione del volgare quale lingua del potere dalla presenza di determinate categorie sociali: da un lato un ceto di laici monolingui (i mercanti), dall'altro una classe dirigente dinamica, in rapporti più o meno conflittuali con il notariato. Tali considerazioni offrono lo spunto per riflettere sul ceto notarile, protagonista indiscusso di lavori di volgarizzamento (sia letterari che documentari), ma che inizialmente sembra ostacolare, a Firenze, l'uso del volgare nei testi di diritto, almeno fino alla sua graduale integrazione nell'amministrazione pubblica comunale.

This essay deals with the use of the vernacular for documentary purposes, in the transmission of normative texts (mainly statuti) between the thirteenth and fourteenth centuries. The author discusses the reasons for choosing the vernacular in specific political and social contexts of thirteenth- and fourteenth-century Tuscany (first in Siena, then in Pisa and Florence), and the important role played by certain social categories in the institutionalization of the vernacular as a language of power: on the one hand a group of monolingual laymen (merchants), on the other hand a dynamic ruling class who had a more or less conflictual relationship with the notaries. This leads us to reflect upon the notaries themselves, uncontested protagonists of translation work carried out into the vernacular (both of literary and documentary writings), but who, in Florence, initially seemed to impede the use of the vernacular in legal texts, at least until it gradually gained its rightful place in the public municipal administration.
\end{abstract}

Parole chiave: Vernacular, Statuti, Siena, Pisa, Florence, Merchants, Notaries

A differenza degli altri interventi di questa sessione, il mio contributo verterà non su testi di natura letteraria o religiosa, ma su documenti di collettività e poteri pubblici, in particolare sul significato dell'uso del volgare delle fonti

Lorenzo Tanzini, Università di Cagliari

Ә Open Access. (C) 2021 Lorenzo Tanzini, published by De Gruyter. (cc)BY This work is licensed under the Creative Commons Attribution 4.0 International License. 
normative della Toscana del XIII, e soprattutto XIV secolo. Per lo stesso motivo in queste pagine incontreremo pochi personaggi o individui riconoscibili - anche se almeno su qualcuno potremo dire qualcosa - e molti soggetti collettivi, i cui intenti si traducono nell'uso della lingua vernacolare. In ogni caso, credo che la differenza di tipologie testuali sia tutt'altro che un impedimento. Anche il volgarizzamento di testi letterari rispondeva ad un intento di 'spiegazione' e di accessibilità più che a quello di traduzione in senso moderno, e quindi nasceva in circostanze simili a quelle di versioni volgari di leggi scritte in latino, che si intendeva far comprendere al di fuori dell'ambito dei colti. ${ }^{1}$ Dall'altra il profilo delle fonti statutarie del XIV secolo non è certo quello di testi rivolti soltanto ad un uso pratico, anzi in certi casi si tratta di elaborazioni che in alcune parti tradiscono un livello culturale ragguardevole, e una circolazione intensa di motivi retorici da fonti di cultura 'alta'. Ciò non può d'altra parte stupire se si pensa che all'uso del volgare in ambito giuridico, nella forma del volgarizzamento, mettono mano essenzialmente notai, cioè la medesima categoria (e talvolta le medesime persone) impegnata in tante imprese di volgarizzamento letterario.

Quale punto di partenza vale la pena ricordare il dato più ovvio per la cultura giuridica bassomedievale, cioè che in linea di principio i documenti si redigono in latino, perché il latino è la lingua del diritto, l'unico codice linguistico che sia sufficientemente raffinato e condiviso per assumere la chiarezza necessaria ai concetti di natura giuridica. ${ }^{2}$ Il contesto italiano da questo punto di vista è nel panorama europeo quello in cui l'equazione testi giuridici = latino si trova applicata con più nettezza e per un tempo più lungo. Nulla di simile alle compilazioni giustinianee provenzali, né alle rielaborazioni del diritto consuetudinario francese o tedesco si trova in Italia, dove di fatto questo monopolio del latino continua fino al Seicento del celebre Dottor volgare di Giovanbattista De Luca. ${ }^{3}$ Questa particolarità italiana introduce un primo paradosso della figura del notaio: sappiamo bene che sono in larga parte notai i protagonisti dei lavori di volgarizzamento, di testi letterari e a maggior ragione di documenti normativi, ma allo stesso tempo è proprio il primato notarile nella redazione

1 Cornish, Vernacular translation, p. 6. Sugli obiettivi (e le difficoltà) del trasferire i contenuti e le modalità espressive della scrittura latina in volgare si veda ora anche Adamska, L'Ars dictaminis.

2 Fiorelli, La lingua del diritto parla, per la grande esplosione di cultura giuridica nel XII secolo, di «un rinascimento senza volgare».

3 Riprendo qui alcune delle considerazioni di Salvestrini, Tanzini, La lingua della legge; per la segnalazione dei singoli casi Fiorelli, Gli "Ordinamenti di giustizia” e Fiorelli, Intorno alle parole del diritto. 
dei documenti che comporta la più durevole resistenza alla penetrazione del volgare nell'ambito del diritto.

L'altro elemento di partenza da enunciare riguarda le tipologie di testi a cui faremo riferimento. Abbiamo accennato genericamente a 'documenti', che tuttavia intenderemo qui come la categoria di testi qualificati come tali nel corpus TLIO, che include come noto formulari, lettere o bolle tradotte e in volgare, e soprattutto statuti. ${ }^{4}$ Agli statuti quindi riserveremo un'attenzione particolare, non soltanto perché rappresentano di gran lunga la categoria più numerosa, ma anche perché hanno il vantaggio di offrirci entrambi i casi possibili, cioè quello dell'uso diretto del volgare nella redazione originaria e quello del volgarizzamento.

\section{Perché il volgare?}

In prima battuta ci dobbiamo chiedere perché, stante il contesto appena enunciato, un certo numero di testi normativi prevalentemente toscani del Due-Trecento siano stati redatti o tradotti in volgare. Le risposte esplicite che talvolta vengono dalle fonti del tempo sono in una certa misura fuorvianti: l'intento di mettere a disposizione uno scritto accessibile a chiunque, orgogliosamente ostentato in più occasioni, non è affatto una risposta plausibile, specialmente considerando l'effettiva circolazione dei documenti in questione. ${ }^{5}$ Quello che conta sono invece i contesti sociali, le effettive situazioni pratiche nelle quali l'uso della lingua vernacolare si rendeva effettivamente necessaria.

In risposta alle esigenze di queste situazioni particolari, la Toscana offre dagli ultimi decenni del XIII secolo una serie di esempi di statuti di comunità che scelgono di affidare al volgare la redazione delle proprie norme. Sono innanzitutto contesti confraternali: a Firenze la compagnia di S. Maria del Carmine $1284,{ }^{6}$ S. Gilio a fine ' $200,{ }^{7}$ Orsanmichele fine ' $200,{ }^{8}$ a Prato quella di S. Croce nel 1295, '

4 Bibliografia dei testi in volgare.

5 Considerazioni illuminanti in proposito quelle di Bambi, Un costituto davvero per tutti? Per le motivazioni espresse dalle fonti del tempo si vedano alcuni esempi sotto, pp. 160-161.

6 Editi in Testi fiorentini del Dugento e dei primi del Trecento.

7 Monti, Le Confraternite medievali, con edizione degli statuti della confraternita fiorentina di

S. Egidio del sec. XIII nel vol. II, pp. 144-158.

8 Nuovi testi fiorentini del Dugento, II, pp. 650-673.

9 Capitoli della Compagnia della Santa Croce di Prato. 
e nello stesso periodo gli statuti dei Disciplinati di Siena ${ }^{10}$ e di Cortona. ${ }^{11}$ Leggermente più tardo è l'esempio di un altro grande soggetto di vita religiosa laicale, lo statuto dello Spedale della Scala di Siena, datato da Luciano Banchi al 1318, ${ }^{12}$ nel quale emerge non solo una versione in volgare ma una vera e propria stesura bilingue: come recita la rubrica CXXIX De scrivere le sopradette constituzioni per gramatica e per volgare, dovranno essere redatti due diversi codici, uno "in grammatica" e uno in volgare, "ad ciò che coloro i quali sanno gramatica e li altri letterati possano avere pieno e chiaro intendimento de le predette cose". Una precisazione indicativa questa perché di senso inverso rispetto a quanto si trova più di frequente negli statuti volgarizzati: il bilinguismo non è pensato a vantaggio di chi non conosce il latino, ma piuttosto per coloro che lo conoscono, affinché l'originaria redazione volgare possa essere presa in considerazione anche dai professionisti del diritto. Ci troviamo dunque di fronte a testi che nascono in volgare, espressione di socialità religiose con una struttura istituzionale labile, che non conoscono un impiego strutturato di notai e quindi tendono a sfuggire a quella equazioni cui si faceva cenno sopra, salvo scegliere in qualche caso di passare al latino come evoluzione verso una forma linguistica più prestigiosa. ${ }^{13} \mathrm{La}$ fattispecie è simile a quella che si riscontra in alcune comunità rurali, specialmente senesi, sempre del periodo di fine '200 e inizi '300. Il caso più clamoroso era stato come noto quello di Montieri, dove si conosce addirittura per il 1219 una redazione volgare preliminare alla stesura in latino. ${ }^{14}$ Nacquero e si sono conservati invece in volgare gli statuti di Montagutolo di Pari (1280-1297) $)^{15}$ Chiarentana $\left(1314\right.$ ? $^{16}$ e Pieve a Molli (1338), ${ }^{17}$ che tuttavia - si badi - vanno intesi come esempi senesi solo nel senso della collocazione geografica, perché nessuna di queste comunità era soggetta a Siena nel periodo dello statuto, e tutte si collocavano invece nel contesto di signorie rurali. ${ }^{18}$

10 Capitoli della Compagnia dei disciplinati di Siena de' secoli XIII, XIV, e XV; si vedano anche Statuti volgari de lo Spedale di S. Maria Vergine di Siena scritti l'anno MCCCV.

11 I Capitoli della Compagnia dei Disciplinati di Cortona (anno 1300).

12 Statuto dello Spedale di Santa Maria di Siena (1318-1379, 1320-1861), II, pp. 73-153.

13 Suggerisce questo tipo di genesi al di fuori della base latina per almeno una parte dei testi qui citati anche Bambi, Alle origini del volgare del diritto, pp. 2-8.

14 Castellani, La prosa italiana delle origini, pp. 41-51, ma si veda Fiorelli, Intorno alle parole del diritto, pp. 80-81.

15 Statuti senesi scritti in volgare ne' secoli XIII e XIV, I, pp. 1-66.

16 In Val d'Orcia nel Trecento. Lo statuto signorile di Chiarentana [1314-1316 ca.].

17 Statuto del Comune della Pieve a Molli del contado di Siena volgarizzato circa l'anno MCCCXXXVIII.

18 Si veda l'ottima sintesi di Dani, Gli statuti dei Comuni della Repubblica di Siena. 
Si possono forse assimilare a queste situazioni di comunità rurali alcuni dei primi statuti in volgare di istituzioni corporative senesi o delle campagne toscane, come gli statuti dei carnaioli, dei cuoiai o dei chiavari di Siena ${ }^{19}$ o quello dell'arte della lana di Radicondoli: ${ }^{20}$ tutti casi nei quali il testo dello statuto non include la nomina di un notaio, quindi non incorpora nelle pratiche consuete della collettività il lavoro di un professionista del diritto.

A conferma di questa funzione soprattutto 'in negativo' della lingua del notaio si può osservare che l'uso esclusivo del volgare ricorre in alcune tipologie istituzionali che sono palesemente, talvolta esplicitamente estranee alle formalità notarili, come il mondo dei mercanti e dei traffici marittimi. Il primato spetta questa volta ai mercanti pisani: il Breve consulum curiae mercatorum, noto in latino nella versione del 1305, ha conservato un volgarizzamento del 1321 con successive aggiunte; mentre il tribunale deputato alla gestione delle controversie marittime, la Curia o Ordine del Mare, fu volgarizzato tra il 1322 e il $1343 ;^{21}$ più avanti nel secolo sarebbero arrivati gli esempi analoghi dei mercanti di Siena e Lucca. ${ }^{22}$ Sempre in volgare troviamo, ai limiti della tipologia statutaria, documenti troppo tecnici per essere redatti in latino come tariffari, libri di gabelle e simili: la gabella delle porte di Siena è la più antica, seguita però da casi analoghi per Pisa e Pistoia nel corso del ' $300 .^{23}$

Ora, questa stagione di volgare senza latino, o di volgare che si pone su binari diversi da quelli della redazione latina, è in realtà tramontata molto presto, e già nel primo '300 di norma troviamo statuti con redazioni bilingui, redatti cioè sicuramente in latino e quindi volgarizzati. Se poniamo la domanda sul motivo di una impresa del genere, credo che l'attenzione debba concentrarsi sui cerimoniali associativi, in particolare tutte le circostanze di lettura pubblica, giuramenti, petizioni in assemblea, nei quali il testo dello statuto più che impiegato professionalmente era fatto proprio dai membri del sodalizio. Come si vede si tratta di un contesto nel quale la parola in volgare rappresenta il versante dell'oralità, una sorta di complemento alla scrittura vera e propria che mantiene il primato nello scritto. Del resto

19 Statuti senesi scritti in volgare ne' secoli XIII e XIV.

20 Statuto dell' Arte della lana di Radicondoli (1308-1388).

21 Statuti inediti della città di Pisa dal XII al XIV secolo, pp. 169-344 e 447-584; la rubrica LXXXVI di quest'ultimo, «De' brevi vulgarmente fare fare e exemplare», disponeva che fossero redatte tre copie della versione volgare, due delle quali destinate agli ufficiali pisani di Cagliari e di Piombino.

22 Lo Statuto dell'Arte della Mercanzia senese (1342-1343); l'edizione è riprodotta in La Mercanzia ad Arezzo nel primo Trecento; Statuto della Corte dei Mercanti in Lucca del 1376.

23 Statuto della gabella e dei passaggi dalle porte della città di Siena; Tanzini, Mercato e fiscalità. 
era questa l'abitudine più tipica del lavoro del notaio, cioè il volgere in scrittura valida in latino ciò che era stato stipulato in volgare, e allo stesso tempo presentare in volgare i contenuti della formalizzazione del latino giuridico. ${ }^{24}$

In questo contesto il passaggio al volgare si lega soprattutto alla pratica di lettura collettiva nell'occasione delle assemblee del sodalizio: lo statuto stesso nella sua versione scritta è in un certo senso redatto in funzione dell'oralità, perché serve in primo luogo come supporto per il rituale della memoria collettiva nella lettura periodica alla presenza di tutti i membri. Lo statuto dello Spedale della Scala deve ad esempio esser letto due volte l'anno nei capitoli semestrali; anni dopo uno degli statuti senesi delle arti di cui si è conservata la redazione volgare, quello dei chiavari, specifica nella rubrica XXXV Ch'e' rectori facciano lèggiare tutto questo breve ogne anno una volta, che

“. . . 'l notaio che le leggiarà debba avere dall'università, per la fatiga de la sua lettura, IIII soldi per ogne volta.”

La lettura in volgare, di nuovo, è pensata come una prerogativa del notaio, che anzi non ha una mansione ufficiale ma viene incaricato - e pagato - espressamente per questo incarico di lettore. E da un notaio veniva effettuata anche in contesti più complessi come nei consigli cittadini. Nel Due e Trecento la dinamica vita politica delle assemblee cittadine si svolgeva come ovvio in volgare, ${ }^{25}$ ma la mediazione tecnica irrinunciabile dei notai faceva sì che le registrazioni scritte delle delibere, e anche dei dibattiti, risultassero immancabilmente in latino. Dal momento però che spesso le stesse norme statutarie imponevano una lettura pubblica di ciò che era stato deliberato, o del parere prevalso in assemblea, ${ }^{26} \mathrm{i}$ notai dovevano essere anche in grado di leggere ai consiglieri testi statutari o modelli di delibere, e i consiglieri dovevano essere messi in condizione di comprenderli: non diversamente del resto di quanto accadeva nel caso di contratti o rogiti notarili. Questa prassi di lettura volgare di statuti e delibere durante le riunioni consiliari è documentata in maniera esplicita in molti casi

24 Emblematico in questo senso il passaggio di Bartolo da Sassoferrato secondo cui "tota die notarii vulgarizant rustico quod est dicto in literali sermone": questo tipo di dinamica fanno riferimento gran parte degli esempi più antichi di testi eminentemente notarili (formulari) tradotti per comodità dei clienti in volgare, sui quali cfr. ora le edizioni e il commento di Bambi, Scrivere in latino; sul notaio e la sua pratica di volgarizzare la parola orale cfr. anche Cornish, Vernacular translation, p. 26.

25 Sottolinea l'esigenza di una formazione alla parola in pubblico anche per i non litterati quale fondamento delle iniziative di diffusione della retorica in volgare Milner, 'Le sottili cose non si possono bene aprire in volgare'.

26 Sulle pratiche di registrazione nelle assemblee comunali cfr. Tanzini, Delibere e verbali. 
del primo Trecento, tra i quali si possono citare i verbali di $\operatorname{Todi}^{27}$ o di Siena. ${ }^{28}$ A Venezia è possibile anche individuare alcuni frammenti statutari in volgare che probabilmente servivano proprio per letture come quelle appena citate, o a situazioni cerimoniali pubbliche quali i giuramenti degli ufficiali. ${ }^{29}$ E' più che plausibile quindi che abbastanza presto siano state realizzate copie d'uso di testi statutari o di schemi di giuramenti da recitare in volgare. Un caso estremo ci soccorre al di fuori dell'ambito toscano. Tra il 1357 e il 1362 venne redatta probabilmente a Perugia la versione volgare delle Costituzioni di Egidio di Albornoz, testimoniata da un unico manoscritto vaticano. ${ }^{30} \mathrm{Si}$ trattava di una operazione certamente impegnativa, che tuttavia rispondeva ad un esplicito impulso dell'autorità pontificia, dal momento che le stesse Costituzioni, al capitolo VI, 27, disponevano al riguardo che i reggitori delle città "ipsas in eorum parlamento publice vel saltem in generali consilio integraliter publicari et exponi faciant in vulgari”. ${ }^{1}$

Questo esempio ci porta a fare un passo ulteriore nella funzione del volgarizzamento. La stesura di un supporto scritto per la pratica di lettura in volgare poteva essere un'esigenza pratica: si potrebbe ricondurre proprio ad una esigenza del genere il caso citato sopra per l'ospedale della Scala, che in effetti aveva un notaio alle sue dipendenze. ${ }^{32}$ Il lavoro di trasformazione di un testo latino di alcune centinaia di carte in volgare andava ben al di là di questo: si trattava piuttosto di una scelta politica, di un investimento che le mere esigenze pratiche del consiglio non avrebbero giustificato. ${ }^{33}$

27 Todi, Archivio Storico del Comune, Riformanze, 9, ff. 44r-44v (1319): convocato il consiglio il difensore proponeva «super supradictis provisionibus hodie factis per XXIIII . . . lectis et vulgariçatis per me notarium».

28 Siena, Archivio di Stato, Consiglio Generale, 109, ff. 130r-130v (1330): «ex forma cuiusdam alterius capituli constituti dicti comunis sub rubrica de consilio fiendo super facto carnificum lecta et vulgariçata per me dominum notarium in presenti consilio».

29 Tomasin, Il volgare e la legge, pp. 33-37, 63-64; a cura del medesimo si vedano anche i Testi padovani del Trecento.

30 Studi sul cardinale Albornoz.

31 Costituzioni Egidiane dell'anno MCCCLVII, pp. 234-235.

32 Come attestato in Statuto dello Spedale di Santa Maria di Siena (1318-1379, 1320-1861), $\S$ CXXVIII.

33 In questo senso mi pare fuorviante, almeno per ciò che concerne i testi normativi, la ricostruzione di Cornish, Vernacular Translation, pp. 24-31, che enfatizza l'importanza delle norme suntuarie, rivolte ad un pubblico 'illitterato' per definizione come quello femminile, come emblema della scelta per il volgare: è vero che la necessità di rendere comunemente comprensibili i termini tecnici della legislazione era una esigenza assai sentita, ma la cronologia dei volgarizzamenti non giunge a suffragare il primato di iniziative del genere, che vengono invece precedute da versioni vernacolari di testi di diritto molto meno tecnici, segno 
Questa vera e propria politica del volgare degli enti pubblici o semi-pubblici ha specifiche cronologie. Indiscusso è il primato senese per gli episodi del primo terzo del XIV secolo, che abbiamo in gran parte già citato, fino allo statuto del 1309-1310. ${ }^{34}$ Pisa ancora nel primo '300 presenta vari casi corporativi o di ambito tecnico mercantile, che non arrivano a coinvolgere le compilazioni più vaste del comune. L'ambiente fiorentino arriva con un certo ritardo. Se si prescinde dal caso dello statuto degli Oliandoli del 1318, i casi noti o effettivamente conservati di statuti redatti in latino e volgarizzati arrivano dagli anni '30: quello non conservato dell'Arte di Calimala 1334, ${ }^{35}$ dei Monetieri nel 1335 o poco prima, ${ }^{36}$ degli Albergatori nel $1338,{ }^{37}$ dei Medici e Speziali $1349,{ }^{38}$ ai quali si aggiungono di nuovo negli anni '30 alcuni volgarizzamenti di ordinamenti specifici del comune, fino ad arrivare allo statuto del 1355. Con gli anni '50 Firenze inizia una campagna per il volgare che si diffonde ovunque, dagli statuti delle comunità soggette ai regolamenti del mercato, fino alle provvisioni aggiunte agli statuti che non hanno neppure un argomento ben definito. ${ }^{39}$ Questo comportava che il lavoro di volgarizzamento finisse per investire anche testi normativi che non avrebbero richiesto un intervento del genere, o magari a versioni in volgare trascurate e approssimative, oggettivamente poco utili anche nel loro effettivo impiego pratico, come è stato osservato ad esempio per la versione volgare dello Statuto della Grascia del $1378 .^{40}$ Anche l'analisi del lavoro di Andrea Lancia sullo statuto fioren-

del fatto che non è principalmente l'intento di comprensione quello che prevale, quanto piuttosto quello di elaborare una lingua per il diritto cittadino e per i valori politici o religiosi fondanti della comunità.

34 Il Costituto del Comune di Siena volgarizzato nel MCCCIX-MCCCX.

35 Fiorelli, Gli "Ordinamenti di giustizia".

36 Constitutum artis monetariorum civitatis Florentiae; la datazione si desume da una nota successiva che fa riferimento all'anno 1335.

37 Statuto degli albergatori volgarizzato; la datazione del testo è quella della versione latina: quanto alla realizzazione di quella volgare si può dire soltanto che fu anteriore al 1370 .

38 Statuti dell'arte dei Medici e Speziali; cfr. § XXX Che i presenti ordini si debbino leggere innanzi all'arte: si prevede la lettura degli statuti a tutti i membri una volta l'anno, ma non si specifica in che lingua. Il testo, tràdito da un manoscritto del XV secolo, presenta un volgarizzamento abbastanza selettivo, che si discosta talvolta dal testo latino del 1349 (presumibilmente perché vuole seguire eventuali modifiche intervenute nel frattempo). L'edizione confonde abbastanza questa situazione dei manoscritti, che pure descrive, perché poi sceglie di pubblicare varie parti prese ora dall'uno ora dall'altro.

39 Le aggiunte alla compilazione statutaria fiorentina; Ordinamenti, provvisioni e riformagioni. 40 Lo Statuto in volgare della Magistratura fiorentina della Grascia (a. 1379). 
tino del $1355^{41}$ sta mostrando come per una serie di aree del testo la versione in volgare si rivelasse una scelta non così efficace quanto si sarebbe potuto pensare proprio per la spiegazione del dettato normativo. Si deve considerare infatti che specialmente nelle materie più pratiche, i regolamenti sul mercato o l'igiene pubblica, lo stesso dettato latino era la trasposizione di contenuti pensati in volgare, o che comunque tradivano la logica del volgare nel lessico e nella sintassi. Dal momento che l'intervento del volgarizzatore, proprio per la sua qualità intellettuale di lettore di testi di cultura, non interveniva a riformulare il dettato latino sul piano giuridico ma aveva un approccio assai letterale, si originava il paradosso per cui il risultato era una sorta di rivolgarizzazione, che portava con sé tutte le imprecisioni e le ambiguità di una traduzione doppia: effetto paradossale perché accentuato soprattutto nelle aree testuali più vicine al volgare. Il caso meriterà ulteriori e più puntuali approfondimenti, ma è comunque significativo per segnalare quanto il lavoro di volgarizzamento rispondesse a scelte che solo in parte erano legate alla mera necessità pratica.

\section{Quali contesti politici?}

Detto questo, sarebbe un errore cercare corrispondenze dirette tra le iniziative di volgarizzamento e i regimi politici nei quali esse si inserirono. Il pragmatismo dei governi cittadini del tempo rende poco verisimile una corrispondenza così ideologica: nel XIV secolo iniziative analoghe possono avere retroterra anche molto diversi, o relazioni ambigue con i rispettivi contesti politici. Ad ogni modo esiste uno sfondo in qualche modo riconoscibile, o quantomeno ci sono circostanze fondamentali che si ritrovano in tutti gli ambienti attivi nel volgarizzamento.

In primo luogo, la presenza di un ceto di laici capaci di leggere il volgare, quindi in linea di massima una presenza degli ambienti mercantili, abituati all'uso della scrittura fuori dagli studi di grammatica, non solo capillare nella società ma anche ben assestata al potere. In secondo luogo, per molti versi corollario della prima condizione, è un ceto dirigente in grado di formulare iniziative non necessariamente ostili ma distaccate rispetto agli ambienti dei professionisti del diritto. Infine, un contesto politico in cui l'elemento della

41 Faccio riferimento qui al lavoro di edizione integrale degli statuti fiorentini del 1355 nel volgarizzamento di Lancia, a cura di F. Bambi, F. Salvestrini e L. Tanzini, in preparazione e previsto in stampa nel corso del 2021. 
partecipazione della cittadinanza alla vita politica è presente come fattore positivo, quantomeno a livello della retorica pubblica, al di là del grado di effettiva rilevanza politica.

Questi elementi si trovano in buona parte dell'Italia comunale, ma l'effetto combinato di tutti e tre contribuisce a stringere il fuoco sulle città toscane.

Vediamo nel dettaglio. Quello della partecipazione è un valore espressamente dichiarato in tutti i casi di volgarizzamenti più impegnativi, laddove $\mathrm{i}$ testi si preoccupino di dichiarare le proprie finalità. Molto noto il caso degli statuti senesi del 1309-1310:

"Et che li signori camarlengo et IIII proveditori del Comune di Siena, sieno tenuti et debiano, sotto pena di X libre di denari per ciascuno di loro, fare scrivere, a l'expese del Comune di Siena, uno statuto del Comune, di nuovo in volgare di buona lettera grossa, bene legibile et bene formata, in buone carte pecorine [. . .] el quale statuto stia et stare debia legato ne la Biccherna, accioché le povare persone et l'altre persone che non sanno gramatica, et li altri, e' quali vorranno, possano esso vedere et copia inde trare et avere a.lloro volontà." ${ }^{42}$

Una versione più essenziale ma altrettanto netta di argomentazioni del genere era nel volgarizzamento dello statuto degli oliandoli fiorentini del 1318:

"a ciò che gli ignoranti e quelli che non sanno la gramatica possino tutti e' capitoli di questo costituto leggere e comprendere volgarmente." ${ }^{43}$

E persino quello dello statuto della Parte Guelfa di Firenze del $1335,{ }^{44}$ un sodalizio che certo non esprimeva idealità proprie della parte popolare della cittadinanza, accennava però ad un simile intento in qualche modo partecipativo

“Anche, acciò che li Statuti de la detta Parte etiandio a' layci siano manifesti; proveduto è che due volumi di statuti presenzialmente si facciano, uno per lectera et l'altro in volgare. Il quale statuto in volgare stare debbia nel palagio de' detti capitani, legato al desco del detto notaio con una catenella, sì che continuamente si possa leggere et vedere.”

Questa retorica della partecipazione presiede non solo alle singole iniziative ma anche alla scelta stessa dei testi da fare oggetto della versione linguistica. A Pisa per esempio nel 1330 si intraprese il volgarizzamento non del Breve del comune, che includeva il diritto applicato nei tribunali e l'architettura istituzio-

42 Il costituto del Comune di Siena volgarizzato nel MCCСІХ-МСССХ, I, dist. I, rub. 134, pp. 122-123.

43 Statuto dell'Arte degli oliandoli. Si noti che nello statuto la rubrica § D’elegere un avicato per l'arte, parla di un "buono experto et savio legista" e la § LXXXI cita del notaio dell'arte.

44 Statuto della parte guelfa di Firenze compilato nel MCCCXXXV; per il volgarizzamento si veda il paragrafo XXXVI. 
nale del governo cittadino, ma il Breve del popolo e delle compagnie, un testo centrato sui meccanismi di partecipazione delle vicinìe all'identità teoricamente popolare del regime. ${ }^{45}$

Questa partecipazione, lo ripeto, vale a livello di discorso pubblico, non necessariamente a metro di una vera e propria natura politica. Come accennato, a Firenze intorno al 1324 si volgarizzano gli Ordinamenti di giustizia, ${ }^{46}$ emblema della legislazione antimagnatizia, e una decina di anni dopo lo Statuto della Parte Guelfa, tradizionalmente appannaggio della parte più oligarchica del ceto dirigente: ma la forza dell'identità guelfa come emblema della città, legata alla tradizione angioina duecentesca ancora viva nell'alleanza con Roberto di Napoli, rendeva lo Statuto della parte un oggetto interessante per una versione volgare. Un caso emblematico di questa scelta di testi con una forte portata simbolica dell'identità cittadina è quello dello Statuto dell'Opera di S. Jacopo di Pistoia. L'Opera era l'istituzione più rappresentativa della religiosità civica della città fin dai tempi più lontani. Lo statuto del 1313 venne volgarizzato da ser Mazzeo di ser Giovanni Bellebuoni, che anni dopo avrebbe legato il suo nome ad una impresa letteraria, il volgarizzamento della Historia destructionis Troiae di Guido delle Colonne del 1333. ${ }^{47}$ Il lavoro di Matteo fu sul piano linguistico molto accurato, ma ebbe durata brevissima, perché lo statuto fu sostituito pochi mesi dopo da una nuova versione latina sotto il dominio di Roberto d'Angiò, espressione della preminenza oligarchica prevalsa sulle istituzioni cittadine. Questa nuova versione latina, però, conteneva forti concessioni cerimoniali al carattere di facciata, demagogico della partecipazione popolare; la nuova versione fu volgarizzata anch'essa, ma in forma abbreviata e approssimativa, tale da configurare più che altro "uno scarno promemoria, oltretutto assai superficiale, del contenuto, con un atteggiamento mentale che denota scarsa considerazione quanto meno per i destinatari": ${ }^{48}$ un tentativo di 'salvare' il contenuto partecipativo del volgarizzamento che tuttavia nei suoi esiti concreti tradisce un carattere strumentale e interessato.

45 Statuti inediti della città di Pisa dal XII al XIV secolo, II, pp. 441-641; si veda il prologo a p. 443: «Questo breve del populo et delle compagne del comuno di Pisa fue translatato et assemprato di gramatica in volgaro del Breve del populo, al tempo delli infrascripti discreti et savi homini honorabili Ansiani del populo di Pisa. Li quali feceno fare la infrascripta opera sì chome di sotto si contiene, adciò che quelle persone che non sano di gramatica, possano avere perfetto intendimento di quelle cose che ne vorrano sapere".

46 Fiorelli, Gli "Ordinamenti di giustizia"; l'edizione del testo si trova in La legislazione antimagnatizia a Firenze, pp. 263-371. Cfr. in particolare S. Diacciati, Introduzione, ibid., pp. XI-XLIII: XXX-XXXI.

47 L'Opera di S. Jacopo. Su Mazzeo, cfr. Pregnolato, Il diritto e la storia in pistoiese.

48 L'Opera di S. Jacopo, p. 120. 
Simili preoccupazioni di richiamare l'elemento della partecipazione non esistono in altri contesti urbani: si pensi a Venezia, dove pure gli esempi di volgarizzamento trecentesco di fonti normative esistono, ma in forma limitata e molto appiattita su esigenze tecnico-pratiche. ${ }^{49}$ Quanto agli altri prerequisiti che abbiamo accennato, vi sono nell'Italia comunali altri esempi illustri di città con una forte tradizione partecipativa, ma nelle quali la forza del ceto dei professionisti del diritto anche nell'autorappresentazione municipale si rifletteva in un ritardo nell'uso del volgare in ambito normativo. Caso emblematico quello di Bologna, dove canale alternativo al notariato non c'è, e in effetti continua l'uso della redazione di statuti in latino anche in ambito corporativo. ${ }^{50}$ Allo stesso tempo Perugia ci ha lasciato una grande testimonianza di volgarizzamento dello statuto cittadino nel $1342,{ }^{51}$ ma sappiamo che l'iniziativa incontrò forti resistenze, dal momento che nel 1324 era comparsa una delibera del comune contro l'idea stessa di scrivere lo statuto in volgare; e in ogni caso non ebbe grande seguito nella pratica delle istituzioni. ${ }^{52}$

L'ostilità verso i notai, o la volontà di creare un canale alternativo, è esplicita e davvero impressionante nel caso senese. Qui la concentrazione degli esempi di volgarizzamento negli anni del Nove non può non tradire una spiccata progettualità politica. Quello del 1309-1310 è uno statuto che si vuol affrancare dalla funzione stessa della cultura notarile così come della mediazione dei professionisti del diritto. ${ }^{53}$ Non a caso nel 1318 si sarebbe assistito a un conflitto aperto della corporazione notarile con il regime senese al governo: la stessa arte venne sciolta e gli uffici pubblici impiegarono notai forestieri, fino a ricomporre il dissidio solo alla metà del secolo. ${ }^{54}$

Una versione in qualche modo depotenziata di questo quadro si trova a Firenze a metà secolo, dove certamente non riscontriamo interventi così ostili al notariato: del resto a Firenze fin dal XIII secolo erano state figure di notai ad avviare e condurre le iniziative di volgarizzamento letterario, animate anche da

49 I Capitolari volgarizzati nel corso del Trecento «nonostante la loro diretta pertinenza alla Cancelleria statale . . . non sono che testimonianze isolate e in ogni caso di dimensioni relativamente ridotte»: Tomasin, Il volgare e la legge, p. 44.

50 Per il caso bolognese si veda ad esempio "Haec sunt statuta": sono volgarizzati gli statuti della società della Seta (1380-1398, n. 24), poi dei drappieri e dei bisilieri (artigiani del lino) nel '400.

51 Statuto del Comune e del Popolo di Perugia del 1342 in volgare.

52 Salvestrini, Tanzini, La lingua della legge.

53 Cfr. Ascheri, Papi, Il Costituto, pp. 9-62, e i saggi raccolti in Siena nello specchio del suo Costituto.

54 Catoni, Il collegio notarile di Siena. 
un deliberato intento di trasmissione di un patrimonio di valori etici ai ceti dirigenti non litterati. ${ }^{55}$ Ciò che riscontriamo a Firenze è comunque una crescente enfatizzazione dell'obiettivo di una giustizia spedita e informale, non ostacolata dalle lungaggini e dalle formalità dei professionisti del diritto, da cui emerge l'opzione per volgare nella pratica della giustizia, ${ }^{56}$ a sua volta in una certa misura assimilabile agli episodi senesi di superamento della mediazione dei professionisti del diritto nelle sfere più delicate della vita pubblica. L'uso del volgare in alcune parti dell'esercizio della giustizia, nelle petizioni degli attori e delle memorie dei comparenti, è abituale: c'era già a Venezia nel tardo '200, ${ }^{57}$ poi si trova nella Corte dei Mercanti di Lucca a metà secolo, e a Firenze nelle corti corporative, tra l'altro anche in quella dell'Arte dei giudici e notai alla fine del secolo. E' invece solo fiorentina la scelta di usare il volgare per tutte le parti del procedimento, compreso il formulario, del Tribunale della Mercanzia, secondo una disposizione del $1355 .{ }^{58}$ La lingua vernacolare entrava quindi a pieno titolo al cuore della pratica del diritto.

Come abbiamo anticipato sopra, nella Firenze della seconda metà del secolo la diffusione del volgare nelle pratiche dell'amministrazione è per certi versi anche più capillare di quanto non fosse accaduto a Siena all'inizio del

55 Come opportunamente ricorda anche il recente contributo di Bischetti, Montefusco, Prime osservazioni su "ars dictaminis", in particolare pp. 177-193.

56 Il contesto del processo vedeva una forma molto intensa di bilinguismo. Nelle cause civili era sempre possibile che fossero prodotti documenti in volgare: specialmente nelle corti mercantili, che accettavano la presentazione di scritture private, di fatto era sulla base di queste che il giudice decideva, e del resto nella prima metà del '300 è abbastanza diffusa la consuetudine di far trascrivere nelle imbreviature notarili interi brani di scritture del genere, che 'pubblicate' in questa forma potevano avere accesso anche ai tribunali più formali. Ma il volgare entrava anche per un'altra via, cioè quella delle testimonianze specialmente nei processi criminali, nei quali si possono trovare inserti di notevole vivacità espressiva. Forse troppo notevole. In certi casi si ha l'impressione che l'impiego del volgare non sia affatto una trascrizione stenografica della testimonianza in una lingua viva e esente dalle rigidità del latino del diritto, ma al contrario sia una vera e propria costruzione a fini narrativi. Si tratta di uno studio ancora da fare, ma l'impressione è che le esigenze di narrazione che emergono nella giustizia criminale finiscano per costituire un quadro fittizio: cfr. per la questione di metodo Zemon Davis, Fiction in the archives.

57 Testi veneziani del Duecento e dei primi del Trecento; rientrano in questa categoria anche gli esempi raccolti nel volume II di Nuovi testi pratesi dalle origini al 1320, pp. 31-323, che contiene estratti in volgare di testi documentari, perlopiù contratti, biglietti con deposizioni in giudizio, spesso cedole in registri pubblici o notarili con sintesi di testamenti o volontà varie. Attestano cioè l'uso frequente del volgare anche nel tardo '200 nei punti di contatto tra l'azione dei privati e il funzionamento della giustizia o delle pratiche notarili.

58 Boschetto, Writing the Vernacular. 
secolo, ${ }^{59}$ ma questa volta una diffusione del genere non avviene contro il notariato, bensì nel contesto di un impiego massiccio di notai al servizio del comune. Quello che stava cambiando era la stessa funzione del notaio nella società: in un contesto nel quale la proliferazione dei professionisti della scrittura ha squalificato socialmente lo stesso status del notaio, l'orientamento prevalente andava verso l'impiego strutturato nelle istituzioni pubbliche. ${ }^{60}$ Una integrazione nell'amministrazione pubblica era ciò che si poneva come prospettiva di ascesa sociale dei notai: questo accentuava a sua volta la tendenza al conformismo e all'identificazione con gli orientamenti del ceto dirigente. Nella stessa Siena del '300 Mathieu Allingri ha recentemente sottolineato proprio questo travaso imponente di esperienze e tradizioni professionali nei ruoli delle istituzioni, soprattutto ruoli subalterni ma dignitosamente retribuiti. ${ }^{61}$ Le esigenze del notariato, che cercava collaborazioni pubbliche come elemento distintivo, si incontravano con questa offerta 'pubblica' per professionisti della scrittura, all'interno della quale stava anche il lavoro del volgarizzamento.

Varrebbe la pena quindi capire il profilo di questi volgarizzatori, un obiettivo per il quale bisogna ammettere che i casi ben documentati sono troppo pochi per anche solo accennare una tipologia. Altre relazioni di questa giornata di studi hanno già affrontato la figura di Andrea Lancia, e ad esse rimando in proposito; quella di Mazzeo di ser Giovanni Bellebuoni conferma i caratteri di un notariato colto, che si muove con disinvoltura tra la pratica giuridica e le letture letterarie. ${ }^{62}$

Il caso più noto è però Ranieri Ghezzi Gangalandi, incaricato del volgarizzamento dello statuto senese del 1309-1310: notaio di varie istituzioni caritatevoli e corporative, uomo organico al regime dei Nove più che rappresentativo della sua corporazione, che infatti non traduce soltanto ma (caso veramente unico) interviene anche nel merito del testo con funzioni di statutario. Ma soprattutto

59 Un secondo momento di grande spinta per il volgarizzamento si colloca dopo il 1419, cioè all'indomani della controversa opera di redazione del nuovo statuto cittadino: a questo torno di anni si ascrivono almeno quattro raccolte di provvisioni ordinate e volgarizzate su singole magistrature o aree tematiche, più il volgarizzamento del V libro (il più corposo) della più recente redazione degli statuti urbani, oggi Archivio di Stato di Firenze, Statuti del comune di Firenze, 32. Si noti che è di poco prima, nel 1414, l'avvio della pratica di redigere in volgare tutti gli atti della giustizia delle Arti, introdotto per volontà del governo del comune ad imitazione di quanto abbiamo già visto sopra per la Mercanzia. Statuti del Comune di Firenze nell'Archivio di Stato; Tanzini, Il governo delle leggi; Boschetto, Writing the vernacular.

60 Si vedano ora i saggi in Notariorum itinera; sull'evoluzione del ruolo dei notai nella società cittadina bassomedievale cfr. Ascheri, I problemi del successo.

61 Allingri, L'activité des notaires siennois.

62 Cfr. nota 47. 
Ranieri è notaio del monastero di San Galgano, la veneranda abbazia cistercense che aveva una sede di grande prestigio anche in città. ${ }^{63}$ La presenza di queste istituzioni religiose 'urbane', i cui membri sono spesso coinvolti nell'amministrazione pubblica per via di incarichi di natura tecnica o contabile, ${ }^{64}$ è un trait d'union sociale e forse anche culturale: negli anni '20 o '30 a Firenze Frate Lorenzo converso della badia di Settimo, al servizio della Camera dell'arme del comune dal 1312 al 1336, volgarizzò alcune rubriche di ambito antimagnatizio che si trovano in latino nello statuto del 1325, "acciò che neuno de' detti priori et gonfaloniere in de le predette cose possa opporre et escusarsi per ignoranza" ${ }^{65}$ Queste figure liminali di laici-religiosi, che partecipano di entrambe le matrici culturali, sono molto ricercate nelle loro funzioni di mediazione linguistica: in fondo non diverso doveva essere l'ambiente per cui lavorò Fantino da San Frediano, copista di uno dei volgarizzamenti del De dilectione di Albertano da Brescia nel $1275 .^{66}$ Si ricordi che già intorno al 1288 era volgarizzato il Reggimento de' principi di Egidio Romano, da un anonimo senese che traduceva dal francese, mettendo a frutto sia la presenza dello stesso Egidio in città in quegli anni, sia la sua amicizia con membri della famiglia Tolomei. ${ }^{67}$ Questi paralleli lasciano intendere che certe istituzioni religiose, con forti legami con il ceto dirigente cittadino e abitualmente frequentate da laici per ragioni di prossimità familiare, di devozione o di governo, potessero essere ambienti preziosi per un travaso di conoscenze e pratiche di lettura da ritrovare nei volgarizzamenti, sia di ambito letterario-religioso che normativo.

In questo modo l'uso del volgare cambiava un'altra volta la sua funzione. Decenni di pratica in città come Siena e Firenze avevano fatto sì che quella vernacolare fosse intesa come la lingua del potere: che si trattasse di una marginalizzazione 'tecnica' del notariato come accade a Siena a inizio secolo, o dell'atteggiamento

63 Neri, Culture et politique à Sienne, pp. 213-217; Neri, Ranieri Ghezzi Gangalandi.

64 Sull'impiego di religiosi nelle istituzioni comunali si veda ora Churchmen and Urban Government.

65 Statuti del Comune di Firenze nell'Archivio di Stato, pp. 252, 262, 508-509, 681.

66 Il trattato della dilezione d'Albertano da Brescia, p. 23: i curatori ipotizzano la committenza da parte di "qualche confraternita o comunità religiosa" fiorentina, anche in considerazione del fatto che il manoscritto giunse alla fine del XIV al monastero del Paradiso, istituzione molto amata dalle grandi famiglie del reggimento cittadino.

67 Il Livro del governamento dei re e dei principi, pp. 25-32. L'edizione dell'opera è a tal riguardo un importante cantiere di ricerche anche per quanto concerne le possibili relazioni con il lavoro di poco successivo per il volgarizzamento dello statuto, che pur avendo finalità (e anche lingue di partenza) diverse si trovava comunque a gestire un lessico di idee e valori politici non lontano. 
meno conflittuale del governo fiorentino, sta di fatto che il volto dell'autorità pubblica parlava sempre più spesso usando il volgare. Questa evoluzione è davvero fondamentale per comprendere un passaggio decisivo della storia della cultura toscana - e con questo torniamo al parallelismo con i volgarizzamenti 'letterari' da cui siamo partiti. Negli stessi anni in cui diventava un topos di trita retorica la ripetizione del volgare come lingua di tutti, quello stesso volgare era diventato essenzialmente la lingua del governo cittadino. In quanto tale veniva esteso, per esplicita volontà del ceto dirigente cittadino, anche a contesto di livello 'alto', ad esempio nell'insegnamento universitario, come ben testimoniano opere come il Paradiso degli Alberti o tutto il mondo che ruota intorno allo Studio fiorentino di fine secolo. ${ }^{68}$ Ben lungi dall'essere una forma 'popolare' di uso della lingua, il volgare si connotava come la lingua del ceto dirigente, anzi della parte più oligarchica di quel ceto. ${ }^{69}$ Le polemiche e i progetti umanistici, le discussioni sull'eccellenza della tradizione letteraria latina o volgare, ${ }^{70}$ non sarebbero state così rilevanti se non si fossero collocate in un assetto di questo tipo.

68 "Utiliter edoceri". Atti inediti degli ufficiali dello Studio fiorentino. Al medesimo ambiente dello Studio, e in particolare al circuito agostiniano fiorentino di S. Spirito enfatizzato nel $\mathrm{Pa}$ radiso degli Alberti, si può ricondurre il volgarizzamento del De civitate Dei, posseduto da Rinaldo Gianfigliazzi, uno dei membri più illustri del reggimento cittadino di quegli anni: cfr. Brilli, Tanzini, Commentare e volgarizzare.

69 Field, The intellectual struggle, in particolare pp. 75-126.

70 Sul ruolo dei volgarizzamenti per la la cronologia e i caratteri dell'Umanesimo a Firenze cfr. Witt, Sulle tracce degli antichi, pp. 179-234, e le riflessioni critiche di Milner, 'Le sottili cose non si possono bene aprire in volgare', pp. 243-244. Ha richiamato con forza questa antichità della congiunzione fiorentina di familiarità coi classici e attenzione alla sfera pubblica Tanturli, Continuità dell'umanesimo civile. 